\title{
Reirradiation for locoregionally recurrent non-small cell lung cancer
}

\author{
Melissa A. L. Vyfhuis, Stephanie Rice, Jill Remick, Sina Mossahebi, Shahed Badiyan, Pranshu Mohindra, \\ Charles B. Simone II
}

Maryland Proton Treatment Center, Department of Radiation Oncology, University of Maryland Medical Center, Baltimore, MD, USA

Contributions: (I) Conception and design: MA Vyfhuis, S Rice, J Remick, CB Simone 2nd; (II) Administrative support: CB Simone 2nd; (III) Provision of study materials or patients: MA Vyfhuis, S Rice, J Remick, CB Simone 2nd; (IV) Collection and assembly of data: MA Vyfhuis, S Rice, J Remick; (V) Data analysis and interpretation: MA Vyfhuis, S Rice, J Remick; (VI) Manuscript writing: All authors; (VII) Final approval of manuscript: All authors. Correspondence to: Charles B. Simone II, MD. Maryland Proton Treatment Center, University of Maryland School of Medicine, 850 West Baltimore St., Baltimore, MD 21201, USA. Email: CharlesSimone@umm.edu.

\begin{abstract}
Locoregional failure in non-small cell lung cancer (NSCLC) remains high, and the management for recurrent disease in the setting of prior radiotherapy is difficult. Retreatment options such as surgery or systemic therapy are typically limited or frequently result in suboptimal outcomes. Reirradiation (reRT) of thoracic malignancies may be an optimal strategy for providing definitive local control and offering a new chance of cure. Yet, retreatment with radiation therapy can be challenging for fear of excessive toxicities and the inability to safely deliver definitive ( $\geq 60 \mathrm{~Gy}$ ) doses of reRT. However, with recent improvements in radiation delivery techniques and image-guidance, dose-escalation with reRT is possible and outcomes are encouraging. Here, we present a review of various radiation techniques, clinical outcomes and associated toxicities in patients with locoregionally recurrent NSCLC treated primarily with reRT.
\end{abstract}

Keywords: Reirradiation (reRT); non-small cell lung cancer (NSCLC); intensity modulated radiation therapy (IMRT); proton beam therapy (PBT); stereotactic body radiation therapy (SBRT)

Submitted Dec 05, 2017. Accepted for publication Dec 08, 2017.

doi: $10.21037 /$ jtd.2017.12.50

View this article at: http://dx.doi.org/10.21037/jtd.2017.12.50

\section{Introduction}

Lung cancer remains the leading cause of cancer death in the United States (US) and worldwide (1). Over $85 \%$ of lung cancers diagnosed in the US are non-small cell lung cancer (NSCLC) and a quarter of patients will present with locally advanced disease (1). Despite improvements in treatment paradigms and advancements in technology, overall survival (OS) for stage III NSCLC remains poor, with 5 -year survival rates ranging from 19-36\% (2). Current National Comprehensive Cancer Network (NCCN) guidelines advocate the use of concurrent chemotherapy and definitive doses of radiation therapy (CRT) for the majority of locally advanced NSCLC patients. Although patients treated with CRT for stage III NSCLC often fail distantly, local failure rates in the thorax can be as high as $30-50 \%$ at 2 years (3-6), with a $25 \%$ risk of isolated locoregional recurrences (7).

Local control in NSCLC is critical and directly impacts OS. A study done by Machtay et al. reviewed prospective data from seven Radiation Therapy Oncology Group (RTOG) trials and found that with every 1-Gy BED (biologically effective dose) increase in dose given to stage III NSCLC patients, there was a $4 \%$ relative increase in survival (6). In a noteworthy meta-analysis examining six randomized trials to determine the benefit of concurrent over sequential CRT, concurrent CRT had a $4.5 \%$ absolute benefit in OS. That analysis also revealed a strong correlation between local control and OS, with no difference in metastatic rates in these stage III NSCLC patients (4). Therefore, maximizing locoregional control in stage III NSCLC can translate into an OS advantage, particularly if the tumor recurs in or near prior radiation 
fields with no evidence of distant metastatic disease.

Treatment of locally recurrent NSCLC after definitive doses of radiotherapy (RT) is challenging. Chemotherapy used as monotherapy in recurrent NSCLC generally shows poor response rates, with progression-free survival (PFS) of only about 4-6 months $(8,9)$, and while immunotherapy following recurrence is an exciting newer treatment strategy, response rates for NSCLC to immune checkpoint inhibitors are limited in the recurrent setting (10). Consequently, alternative methods are often considered as a means to attain durable locoregional control, including surgery or reirradiation (reRT). Surgical resection after definitive CRT as part of trimodality therapy is feasible only in carefully selected patients with stage III NSCLC $(11,12)$, but it is not routinely pursued after high ( $\geq 60 \mathrm{~Gy})$ doses of neoadjuvant CRT (3) and is most optimally performed within $8-10$ weeks of completing RT given the increasing difficulty and morbidity of thoracic surgery after this time period (13-16). Similarly, radiation oncologists often hesitate to reirradiate after definitive CRT beyond palliative doses due to concerns of excess toxicities to surrounding organs-at-risk (OARs), which include the heart, lungs, spinal cord and esophagus $(17,18)$.

However, with recent advances in external beam radiotherapy (EBRT) delivery, dose escalation of reRT treatments have been possible (19-24) with increased conformality and a decrease in dose to surrounding OARs. The focus of this review article is to summarize studies published that report on clinical outcomes and treatment-related toxicities in patients with NSCLC who developed locoregionally recurrent disease and were retreated predominantly with RT. The benefits of intensitymodulated radiation therapy (IMRT), proton beam therapy (PBT), and stereotactic body radiotherapy (SBRT) over 3D-conformal radiotherapy (3D-CRT) techniques will also be discussed, as well as future directions to improve clinical outcomes in this patient population.

\section{Conventional photon reRT therapy}

Early reRT studies in NSCLC utilized conventional (2D) or 3D-CRT delivery techniques and seldom provided treatment beyond palliative doses (up to $30 \mathrm{~Gy}$ ). Outcomes were poor with a median survival of approximately 5 months (Table 1). Some institutional experiences whose median reRT dose was $>30-35$ Gy, resulted in an observable improvement in survival using 3D-CRT. For example, $\mathrm{Wu}$ et al. reported on a prospective phase I-II study at the Fudan University in China. The majority of patients were initially diagnosed with either stage II $(n=7)$ or stage III $(\mathrm{n}=16)$ NSCLC and were originally treated with a median dose of 66 (range, 30-78) Gy. ReRT doses (range, 46-60 Gy) were chosen based on the first course of radiotherapy; if patients received $>50$ Gy primarily, then 46-50 Gy was given as a reRT dose at 2 Gy per fraction, with sequential chemotherapy only offered to patients with a good performance status (23). While maintaining strict OAR constraints during their retreatment plan optimization (i.e., spinal cord <25 Gy, minimizing lung V20), they managed to observe a locoregional progression free survival of $52 \%$ at 1 year and a median survival of 14 months, nearly 3 times longer than what was seen with palliative reRT doses delivered in earlier studies (Table 1). Toxicity was also minimal, with no grade 3 or greater acute toxicity reported at last follow up (23).

While 3D-CRT uses patient-specific geometry as a means to deliver RT, limited beam arrangements and a uniform dose in each beam results in simple, large fields with higher doses to OARs and subsequently more toxicity compared to more conformal techniques (25-28). IMRT is now a more common method for delivering radiation for thoracic malignancies. Unlike 3D-CRT, treatment plans using IMRT are inversely optimized generally to deliver a more conformal dose distribution to the tumor along with a sharper dose falloff, thus typically sparing highdose radiation to nearby OARs (29). Furthermore, the intensity of each photon beam in an IMRT plan can be adjusted via field modulation using multileaf collimators (MLCs) or through dose-rate alterations (29-34). These treatment characteristics can be desirable in patients with locoregionally recurrent NSCLC, especially if definitive doses of RT were given for the initial course of treatment.

A retrospective study at the University of Wisconsin reported on a total of 37 NSCLC (54\% stage III) patients who developed recurrent disease, the majority of whom (95\%) were retreated using IMRT. Half of the cohort was retreated with palliative intent to a median of 30 (range, 12-60) Gy in the reRT setting, consequently leading to a poor median survival of 5 months (35). On multivariate analysis (MVA), a higher dose at time of reRT $(\mathrm{P}=0.007)$ and performance status $(\mathrm{P}=0.01)$ were associated with improved survival in this cohort (35). One of the largest institutional experiences with reRT of recurrent NSCLC was reported by investigators from MD Anderson, where 102 patients underwent retreatment with highly conformal modern techniques of either IMRT or PBT to definitive doses (19). 


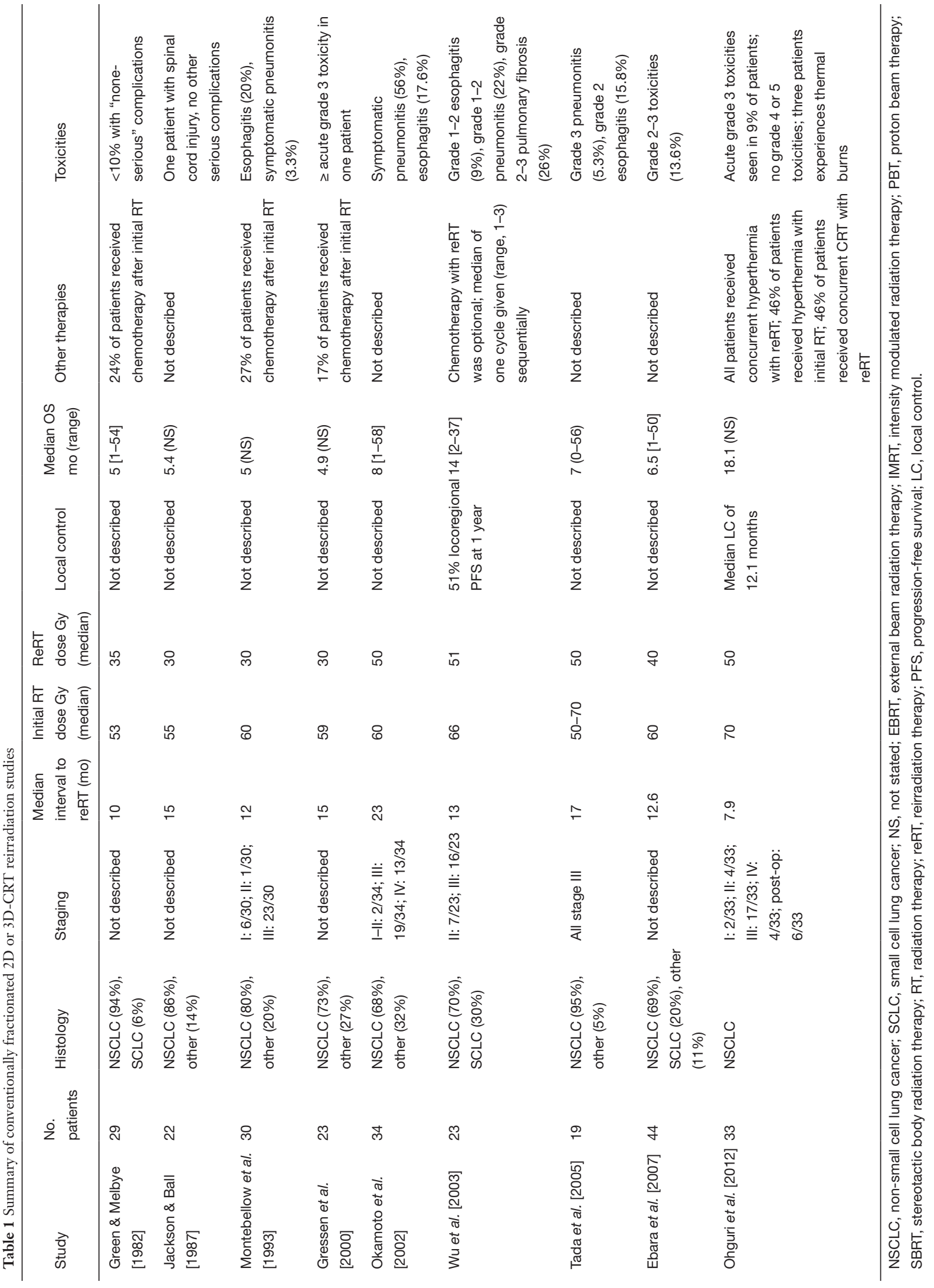


Once again, higher EQD2 dose at time of retreatment was predictive for improved OS on MVA [hazard ratio $(\mathrm{HR})=0.246,95 \%$ CI, 0.075-0.86, $\mathrm{P}=0.021]$ (19). These data suggest that increasing dose of RT in the recurrent setting may improve clinical outcomes in patients with NSCLC. However, one must consider the possible toxicities that may diminish a potential OS benefit with definitive doses of reRT. As such, continued dose reductions to OARs are necessary with newer EBRT innovations.

\section{Conventional proton reRT therapy}

Proton therapy is an ideal treatment modality for reirradiation in NSCLC (36), based upon the physical property of the Bragg peak, where the majority of energy deposition occurs at a set distance with minimal to no "exit dose" beyond this point, thus sparing nearby thoracic OARs (37). There are two predominant types of proton therapy utilized in the US: passive scatter proton therapy (PSPT) and pencil-beam scanning proton therapy, which allows for intensity-modulated proton therapy (IMPT). PSPT uses a $3 \mathrm{D}$ treatment planning technique with the aid of one or two scatterers to expand the width of the proton beam delivery depending upon the width of the target. Following scatter of the proton beam, a range compensator is uniquely made for individual tumors to shape the proton therapy dose to the distal edge of the tumor. In order to shape the beam laterally, an aperture (usually made of brass) is used $(38,39)$. On the other hand, IMPT uses magnets and a narrow proton beam to deliver discrete spots (about 4-9 $\mathrm{mm}$ in diameter) of protons in a 2D-plane, painting the tumor target layer by layer. This method also allows for the modulation of the weight of the individual beamlets in each layer, which gives a higher dose conformality to the target, analogous to IMRT, but with a lower integral dose to nearby OARs $(40,41)$. IMPT does not require the patientspecific aperture or range compensator devices needed in PSPT, often making treatment planning more streamlined.

While IMPT offers more conformal therapy and better OAR sparing, there is increased risk of "interplay effect" with tumor motion, which could lead to over- or under-dosing of targets depending upon intra-fractional tumor motion (42-45) and modulation of the proton beamlets. This "interplay effect" can degrade the quality and robustness of an IMPT plan (42,46-48). Use of 4D$\mathrm{CT}$, repainting techniques, and worst-case-scenario-based optimization are some of the techniques that have been utilized to improve robustness of IMPT thoracic radiation delivery (49-52).

Two early studies by investigators at MD Anderson characterized reRT of NSCLC using PSPT. The first evaluated reRT (20), where a quarter of the patients received concurrent chemotherapy. The median dose of initial radiation was 63 (range, 40-74) Gy, with a median EQD2 of 62.2 (range, 39-155) Gy. The study population received various treatment strategies prior to reRT, including chemotherapy (45\%), surgery $(6 \%)$, or both surgery and chemotherapy (6\%). The majority of the tumors $(85 \%)$ were centrally located, and with a median reRT dose of 66 (range, 16.4-75) Gy (RBE), median OS was 11.1 months, with 6-, 12-, 18-, and 24-month OS of $75 \%, 47 \%, 37 \%$, and $33 \%$, respectively. Grade 3 or greater toxicities were observed in one-third of patients (9\% esophageal, $21 \%$ pulmonary), and no grade 5 events occurred. Administration of concurrent chemotherapy did not improve local control, nor did it increase grade 3 or higher toxicity overall $(\mathrm{P}=0.218)$.

The second MD Anderson analysis included 102 patients undergoing reRT with either IMRT or PSPT to report outcomes, define dose constraints, and provide guidance as to which candidates are optimal for definitive doses of reRT (19). Slightly higher than the prior study, the median EQD2 for initial radiation was 70 (range, 33-276) EQD2 Gy, with a median time to tumor recurrence and reRT of 11 (range, 0-375) and 17 (range, 0-376) months, respectively. Median retreatment dose was 60.5 (range, 25.2-155) EQD2 Gy. At a median follow up of 6.5 months, median OS was 14.7 (range, 10.3-20.6) months. Six, twelve, eighteen and twenty-four months OS were $80 \%, 52.8 \%, 41.4 \%$, and $32.6 \%$, respectively. Only $17 \%$ of patients had any acute grade $\geq 3$ toxicity ( $7 \%$ esophageal, $10 \%$ pulmonary), and concurrent chemotherapy was associated with higher acute grade $\geq 2$ esophageal toxicity $(\mathrm{P}=0.029)$. However, location of tumor, iGTV, ITV, IMRT vs. PSPT, and EQD2 at retreatment were not associated with higher rates of grade 2 or higher esophageal toxicity, and only lung V10, $\mathrm{V} 20$, and mean lung doses were associated with risks of grade 2 or higher pulmonary toxicities. On MVA, receipt of combined modality therapy predicted for better local control (HR =6.48; 95\% CI, 2.28-18.36, $\mathrm{P}=0.0004$ ), as did having greater than a 6-month interval between irradiation courses (HR $=0.374 ; 95 \%$ CI, 0.173-0.806; $\mathrm{P}=0.012$ ). Adenocarcinoma histology (HR $=0.383 ; 95 \%$ CI, $0.2-0.735$; $\mathrm{P}=0.004)$, concurrent chemotherapy ( $\mathrm{HR}=2.613 ; 95 \% \mathrm{CI}$, 1.348-5.066; $\mathrm{P}=0.0045$ ), and higher EQD2 at reRT (HR $=0.246 ; 95 \% \mathrm{CI}, 0.075-0.86 ; \mathrm{P}=0.021)$ all independently 
predicted for OS.

Chao et al. recently reported on a multi-institutional prospective trial of 57 patients with recurrent NSCLC retreated with PSPT or IMPT (10.6\% of patients) (21). With a median time between radiation courses of 19 (range, $3.5-151)$ months, $68 \%$ of patients received concurrent chemotherapy with reRT, and a median reRT dose of 66.6 (range, 30-74) Gy was delivered. Reported toxicities were notable, with grade 3 or higher toxicities (acute or late) occurring in $42 \%$ of patients, including four patients with grade 4 and 6 with grade 5 toxicities. Factors associated with higher rates of toxicities included greater than the median amount of central region overlap (acute grade $\geq 3$ toxicity $64 \%$ vs. $14 \%, \mathrm{P}<0.001$ ) and greater than the median dose to the esophagus (acute grade $\geq 3$ toxicity $64 \%$ vs. $22 \%, \mathrm{P}=0.003$ ) and heart (acute grade $\geq 3$ toxicity $60 \%$ vs. $26 \%, \mathrm{P}=0.02$ ). At a median follow up of 7.8 (range, 1-40) months, median survival was 14.9 months. Although central overlap was associated with increased acute toxicity, decreased overlap did not translate into an OS benefit $(63 \%$ vs. $55 \%, \mathrm{P}=0.3)$. Similarly, lower mean heart dose $(59 \%$ vs. $57 \%, \mathrm{P}=0.8)$ and concurrent chemotherapy (66\% vs. $43 \%$, $\mathrm{P}=0.3$ ) did not translate to increased OS. However, patients with a lower mean esophageal dose did have significantly improved 1-year OS when compared to those with higher mean esophageal dose ( $74 \%$ vs. $38 \%, \mathrm{P}=0.007)$.

Most recently, Ho et al. has published the first IMPT reRT series on 27 patients treated with definitive retreatment doses in the thorax (53). The majority of patients $81 \%$ (22/27) had NSCLC histologies, and prior retreatment in this series included 2D, 3DCRT, SBRT, or proton radiation. Most patients $(85 \%)$ had their prior radiation fields overlapped within the $100 \%$ isodose line of the retreatment plan, and $81 \%$ of patients had centrally located tumors. The median time between initial radiation and reRT with IMPT was 29.5 (range, 0.1-212.3) months, and the median prior RT dose was 60.0 (range, 36-226.8) EQD2 Gy. The median reRT dose given was 66 (range, 43.2-84) EQD2 Gy and 48\% (13/27) of patients underwent concurrent chemotherapy, most commonly with carboplatin and paclitaxel. At a median follow up of 11.2 months, median OS was 18.0 months, with 6-, 12-, and 18-month OS of $89 \%, 54 \%$, and $54 \%$, respectively. reRT in this series was well tolerated, with only $7 \%$ of patients developing a grade 3 pulmonary toxicity and no grade 4 or 5 toxicities recorded (Table 2).

A recent study utilizing the Proton Collaborative Group (PCG) prospective database reported on reRT outcomes in 67 patients who were primarily diagnosed with NSCLC $(n=60)$. The majority of these patients received chemotherapy $(86 \%)$ prior to reRT and $30 \%(n=20)$ received concurrent chemotherapy with retreatment, with a median reRT dose of 60 (range, 30-74) Gy. Median survival for the entire cohort was 13.2 and 14.2 months for those treated definitively. Toxicities in this series seemed manageable with only $3 \%$ of the patients experiencing an acute grade 3 toxicity (pneumonia and neck pain) and 1\% having late grade 3 fatigue. There were no acute or late grade 4 toxicities observed and one patient died 4.5 months after reRT of an unclear cause (54).

Proton therapy is an ideal treatment modality for reRT in NSCLC patients, potentially allowing for full dose ( $\geq 60 \mathrm{~Gy}$ ) retreatment to enhance local control while mitigating toxicities through a decrease in integral dose to surrounding OARs, all of which may lead to improvements in OS. Although most reports to date are retrospective experiences, the above studies collectively suggest that more conformal radiation techniques using proton therapy (i.e., IMPT) could afford better normal-tissue sparing and could possibly improve clinical outcomes. Future studies should be aimed at the evaluation and inclusion of IMPT for patients requiring thorax reRT, which can allow for safer dose escalation and utilization of concurrent CRT, with a theoretical improvement in toxicity when compared to other conventional EBRT techniques.

\section{reRT using SBRT}

Stereotactic body radiation therapy (SBRT), also termed stereotactic ablative radiotherapy (SABR), is a highly conformal radiation technique that delivers a high dose of radiation per fraction that can treat various tumors throughout the body. SBRT for the primary treatment of early stage NSCLC in medically inoperable patients is wellestablished and results in excellent local control with a low toxicity profile (55-59).

With the use of SBRT, a higher biologic effective dose (BED) to the tumor has also been indicated to improve outcomes $(56,60,61)$. Onishi and colleagues demonstrated a decrease in local failure ( $8 \%$ vs. $26 \%$ ) and improved OS (70.8\% vs. 30.2\%) among early stage NSCLC patients who received a $\mathrm{BED} \geq 100$ Gy compared to a BED $\leq 100$ Gy (56). A meta-analysis by Zhang et al. subdivided BED into quartiles and found patients who received a BED ranging from 83.2-146 Gy also had improved OS (60). Additionally, higher BED regimens have also correlated with excellent 


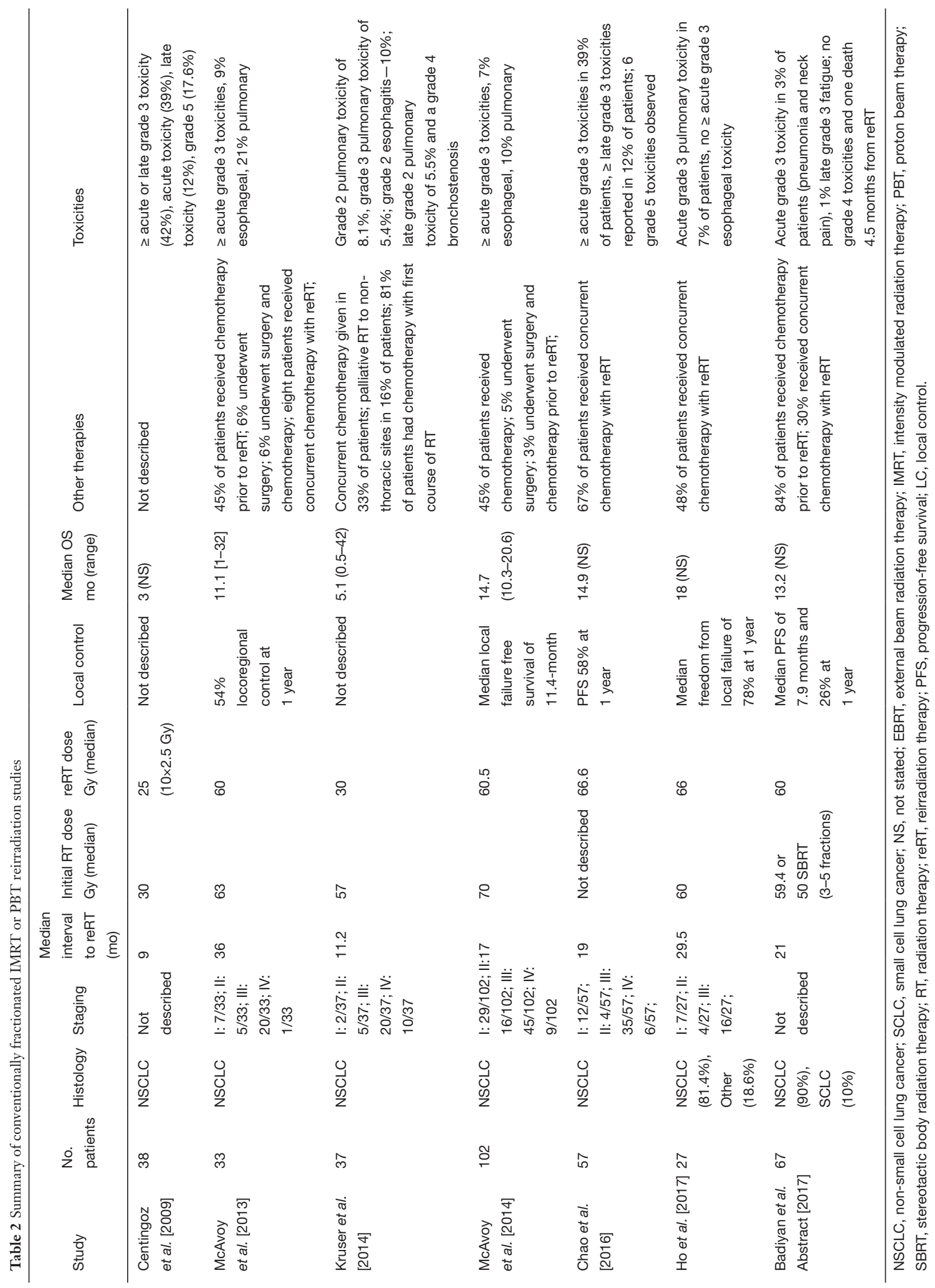


local control rates in the treatment of oligometastatic disease $(62,63)$. Unlike conventional photon fractionation, SBRT delivers ablative doses to the tumor with a steep dose falloff, therefore, protecting OARs from high doses of radiation (64). In the treatment of early stage lung cancer, SBRT can significantly reduce the rate of esophagitis, pneumonitis and dyspnea, and also significantly improve quality of life (QoL) compared to conventionally fractionated regimens (65).

The improved therapeutic ratio that can be achieved with SBRT is particularly appealing in the reRT setting, where minimizing dose to previously irradiated normal tissue is critical. There have been several small studies that have assessed the efficacy and safety of SBRT with promising results (Table 3). Overall, clinical outcomes appear to be favorable, with local control rates ranging from 50-95\% and median survivals ranging from 14-40.9 months (Table 1) (66-77). These results are encouraging when compared to historic, palliative outcomes using conventional radiation, as noted previously $(23,24)$.

In concordance with SBRT for early stage lung cancer, higher BED appears to correlate with improved local control, as was demonstrated by a report from investigators at MD Anderson $(67,70)$. In that report, local control was $92 \%$, and upon further analysis of patients who received optimal BED ( $\geq 100$ Gy) without compromising PTV coverage, local control was $96 \%$ with a 2 -year OS of $59 \%$ (67). An updated analysis of a larger cohort of patients reported local control that exceeded $95 \%$ and 2 -year OS of $74 \%$ after SBRT reRT most commonly to $50 \mathrm{~Gy}$ in 4 fractions (70). Of note, only $30 \%$ of these patients were treated for an in-field relapse as defined by the target within the $30 \mathrm{~Gy}$ isodose line of the prior field. A study by Killburn et al. specifically addressed outcomes in patients with in-field relapses and reported a 2 -year LC of $67 \%$ with a prescription BED of 75 Gy (50 Gy in 10 fractions) delivered for the majority of cases (69).

Higher BED has also been associated with improved survival using SBRT in the reRT setting. Reyngold et al. analyzed 39 patients with a prior history of conventional radiotherapy to the thorax (74). They delivered a median BED of 48 Gy in patients who had overlap of their prior radiation field $(\mathrm{n}=22)$, compared to $106 \mathrm{~Gy}$ when there was no overlap (17). With a median follow up of 12.6 months, local-progression free survival (LPFS) was $64 \%$ at 2 years and the median OS was 22 months. Notably, patients treated with a BED of $\geq 100$ Gy had improved LPFS, recurrence free survival and OS (74). Similarly, Parks and colleagues reported the results of 29 patients, where $45 \%$ $(\mathrm{n}=13)$ had in-field failures and $59 \%(\mathrm{n}=17)$ were centrally located (72). A BED $\geq 100$ Gy was delivered to 20 patients (69\%). They reported a median survival of 40.9 (range, 4.6-77.1) months, with a 2 -year OS of $65 \%$. There was a significant improvement in 2-year survival among patients that received $\mathrm{BED} \geq 100$ Gy compared to those who received $\mathrm{BED}<100 \mathrm{~Gy}, 91 \%$ vs. $55 \%$, respectively. This is similar to the findings of Seung et al., in which 7 out of 8 of the patients received $\mathrm{BED} \geq 100 \mathrm{~Gy}$, all of which were alive with a median follow up of 18 months, and they had a local regional control of $86 \%$ (75).

Despite the dosimetric advantages that SBRT can offer in a reRT scenario, retreatment toxicity can be severe, particularly when delivered to tumors near central structures like the bronchial airways. Dose escalation studies have guided our understanding of maximum-tolerated dose for both conventional radiotherapy and SBRT treatment, respectively $(5,78)$. However, large prospective studies to assess the safety and efficacy of SBRT in the reRT setting are lacking, and dosimetric data are not consistently reported. In select studies in which high median BED was delivered ( $\geq 100 \mathrm{~Gy}$ ), the rate of grade 3 toxicities range from $5-33 \%$, with pulmonary toxicities being the most common $(67,70,77)$. A Swedish study reported a $48 \%$ incidence of acute grade 3-4 toxicities, including $10 \%$ grade 5 toxicities, in which all grade 4-5 toxicity (5) events occurred in centrally located tumors (68). Trovo et al. assessed outcomes of centrally located recurrences using a dose of 30 Gy delivered in 5-6 fractions and reported a 23\% incidence of grade 3 pneumonitis and $12 \%$ incidence of grade 5 toxicity (76). Parks et al. treated 17 centrally-located tumors in their cohort of 29 patients and although they did not report any grade 4-5 toxicities, the rate of grade 2-3 pulmonary complications was $63 \%$ (72).

In the MD Anderson experience, investigators reported a $19 \%$ incidence of grade 3 radiation pneumonitis (RP) (67), and the incidence of RP was significantly associated with out-of-field relapse but did not correlate with tumor size, location, SBRT dose or interval from prior treatment. In their updated report, they found pre-SBRT performance status, FEV $1 \leq 65 \%, \mathrm{~V} 20 \geq 30 \%$ in the combined plan, and previous bilateral mediastinal PTV predicted for the development of grade 3-5 RP (70). They determined that the composite plans (initial RT course + reRT dosimetry) were the most predictive of RP incidence. A scoring system was developed based on these factors to potentially identify patients that would benefit most from reRT (70). In contrast, 


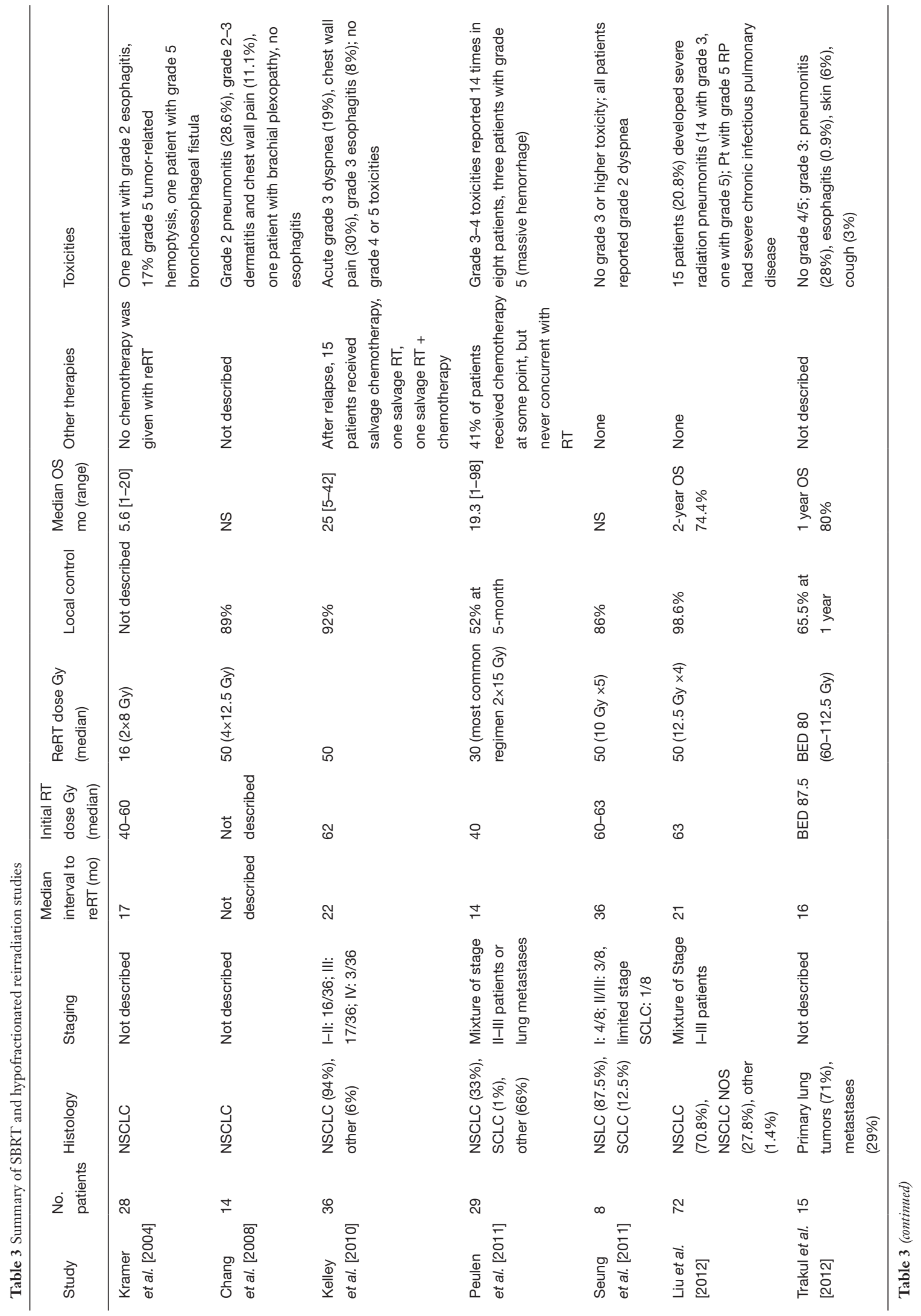




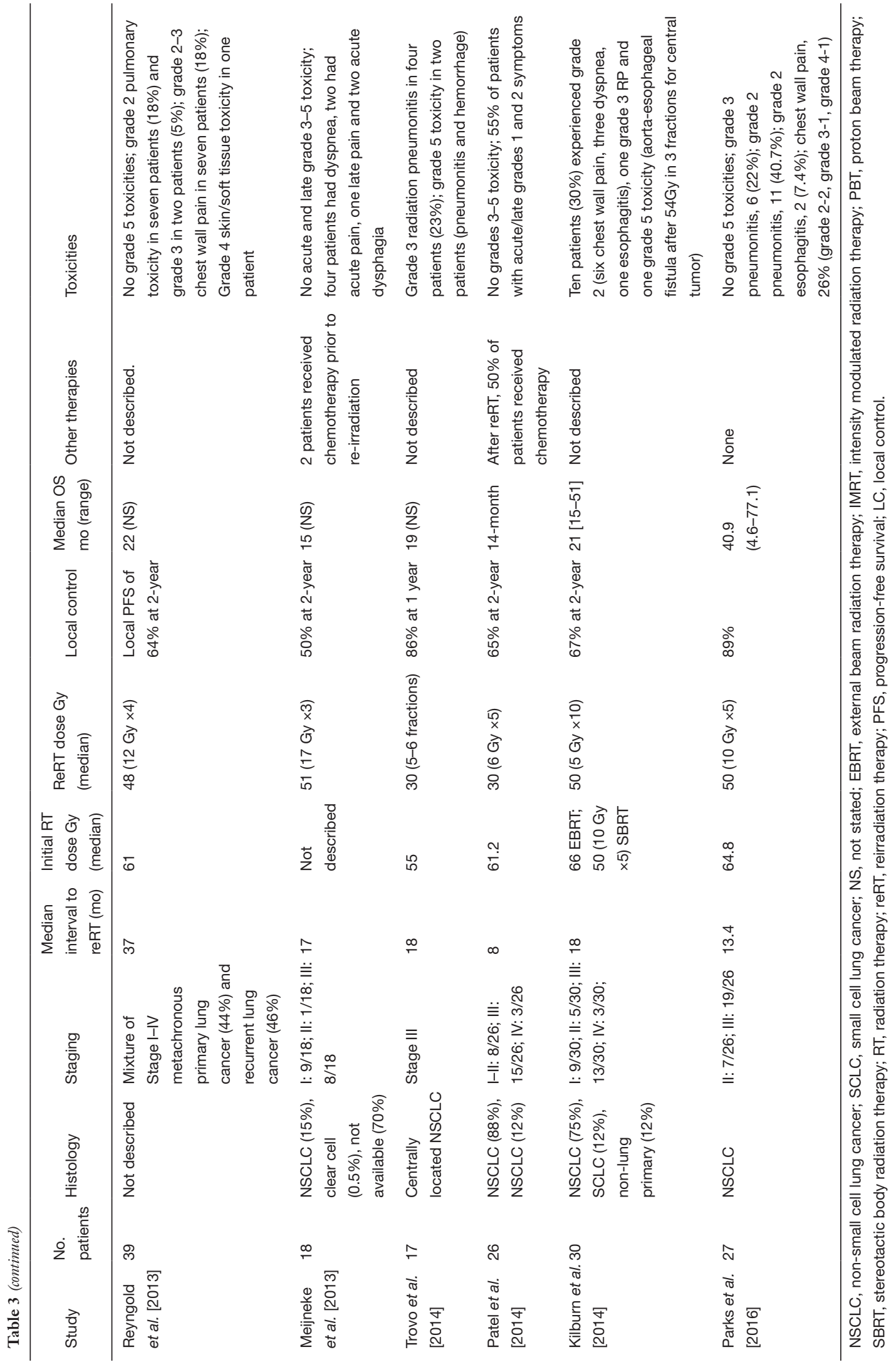


Kilburn et al. reported a reasonable toxicity profile among 33 patients treated with SBRT, where only 30\% experienced a grade 2 toxicity and one patient a grade $3 \mathrm{RP}(69)$. A more conservative dose of $50 \mathrm{~Gy}$ in 10 fractions was the most common regimen given in this experience since the majority of patients were treated for an in-field recurrence. They reported one grade 5 event resulting from exsanguination of an aorta-esophageal fistula after $54 \mathrm{~Gy}$ in 3 fractions to a centrally-located tumor in which the aorta was within the $100 \%$ isodose line for both treatment plans (69).

Data reported using SBRT in reRT NSCLC are limited to retrospective experiences, small patient numbers, short follow-up times and a fairly heterogeneous patient population. Additionally, dosimetric and clinical details are not consistently reported, which makes definitive retreatment guidelines challenging to develop. However, these studies have suggested several clinical parameters that should be considered to aid in identifying ideal patients for reRT using SBRT. Factors such as performance status, pretreatment lung function $(70,74)$, smaller PTV volume (74) and a BED >100 Gy $(72,74)$ have been predictive of improved outcomes. SBRT reRT is a reasonable salvage option in well-selected patients with a history of prior thoracic radiation treatment and can be associated with high rates of local control and favorable survival in comparison to palliative doses of conventional radiation therapy. However, treatment-related toxicities can be severe, especially in centrally-located recurrences $(68,76,79)$ and, therefore, careful patient selection is critical.

\section{Discussion}

There have been many studies looking at the feasibility and safety of reRT in recurrent NSCLC; however, the majority of these experiences using conventional photon therapy have prescribed palliative retreatment doses, resulting in poor survival and local control. 3D-CRT has been shown to be an excellent choice for palliative reRT in patients with recurrent NSCLC, providing symptomatic relief in approximately $70-80 \%$ of cases with a low rate of retreatment toxicities $(13,18,80,81)$. However, in the context of definitive retreatment, increasing reRT dose can potentially improve OS and offer a chance of cure, particularly in patients with limited locoregionally recurrent disease $(19,35)$. While a higher BED can conceivably improve local control and OS in the initial diagnosis of NSCLC (6), elevated doses in the reRT setting are perhaps more important given the hypoxic conditions generated after prior treatment leading to more radioresistant tumors (82).

Advancements of EBRT delivery and improvements in image-guidance have allowed radiation oncologists to feel more comfortable delivering definitive doses of reRT ( $\geq 60$ Gy) for recurrent NSCLC. The MD Anderson series $(19,20)$ utilized IMRT or proton therapy to a median retreatment dose of $60 \mathrm{~Gy}$ in recurrent NSCLC patients and had comparable treatment-related toxicities to older series that only used palliative doses, while improving median survival by 3 -fold. In the Chao et al. study, longterm survival was also achieved, although nearly two-thirds of their patients received concurrent chemotherapy with their reRT (median, 66.6 Gy), which expectantly had an increase rate of toxicity (21). Concurrent CRT can possibly improve survival in the recurrent NSCLC setting (19), and further studies are needed to confirm the benefit of concurrent over sequential CRT in the recurrent setting. Furthermore, dose to the heart, esophagus and lungs are all important and correlate with toxicities in the more recent reRT studies (19-21); thus, further dosimetric improvements are necessary. The majority of the proton therapy experiences in the reRT NSCLC setting have used PSPT technology as mentioned previously (19-21). IMPT provides a dosimetric advantage over PSPT and IMRT $(32,41,51,83)$ and over PSPT (84) which can potentially reduce toxicities associated with reRT in the thorax that may in turn lead to improvements in clinical outcomes (53). Indeed, the only published institutional experience using IMPT comes from MD Anderson, and although such a retrospective study is subject to selection bias with a fairly heterogeneous patient population, a median survival of 18 months in recurrent NSCLC using conventional fractionation should stimulate the development of future treatment studies (53).

SBRT has also been shown to be an effective retreatment strategy in NSCLC, particularly in patients with peripherally located lesions. Local control rates using a higher dose per fraction in patients with recurrent NSCLC disease are impressive, ranging from $60-90 \%$ in most studies. However, the location of disease recurrence plays a critical role in the likelihood of development of treatment-induced toxicities after reRT with SBRT. SBRT for centrally located tumors near critical airway structures can lead to excessive toxicity and mortality $(78,85)$, a trend that continues to be important in the reRT setting $(68,86)$. As noted by Peulen et al., $34 \%$ of patients had centrally located recurrences, and this subset composed all grade 4-5 toxicities in their experience. Interestingly, the most common dose per fraction 
in their study was $15 \mathrm{~Gy}$, which could have contributed to the morbidity and mortality seen (68). However, even when utilizing more fractionated SBRT regimens that have been deemed safe in the de novo setting for centrally located early stage NSCLC tumors (78), there remains a severe toxicity concern associated with retreating these tumors with a hypofractionated approach (76). Hence, for the majority of centrally-located recurrent cases, conventional fractionation or a more mild ( $\geq 8-10$ fractions) hypofractionation paradigm should be considered.

Although there have been improvements in radiation delivery and precision, with any NSCLC locoregional recurrence, there is always a concern for distant failures. In that regard, there have been a number of systemic advances in NSCLC, especially through the use of immunotherapy (10,87-89). Nivolumab and pembrolizumab, both of which are programmed cell death receptor-1 (PD-1) inhibitors, have been shown to improve PFS and OS in patients with progressive NSCLC as a second-line systemic options over single-agent docetaxel $(87,88,90)$. Pembrolizumab was also found to be superior to other chemotherapeutic agents as first-line treatment in metastatic NSCLC (10). The synergistic potential between RT and immunotherapy $(91,92)$ is actively being investigated for lung cancer (93-95). Currently, there are no open clinical trials in the US looking at the combination of RT and immunotherapy in recurrent NSCLC, but such a novel approach is sure to be investigated in future studies.

\section{Conclusions}

Locoregional recurrences remain common in patients with locally advanced NSCLC, and reRT options have historically been limited to palliative doses. Modern RT techniques have allowed for dose-escalated, definitive doses of reRT to be safely given in select patients with recurrent disease and resulted in improved clinical outcomes. Nonetheless, with definitive retreatment in the thorax comes the risk of significant toxicities, and patient selection is critical in order to maximize the benefits of reRT. Prospective clinical studies are needed to optimize patient selection and to facilitate the integration of these different radiation modalities into the management of locally recurrent lung cancer.

\section{Acknowledgements}

None.

\section{Footnote}

Conflicts of Interest: The authors have no conflicts of interest to declare.

\section{References}

1. Siegel RL, Miller KD, Jemal A. Cancer Statistics, 2017. CA Cancer J Clin 2017;67:7-30.

2. Goldstraw P, Chansky K, Crowley J, et al. The IASLC lung cancer staging project: Proposals for revision of the TNM stage groupings in the forthcoming (eighth) edition of the TNM Classification for lung cancer. J Thorac Oncol 2016;11:39-51.

3. Albain KS, Swann RS, Rusch VW, et al. Radiotherapy plus chemotherapy with or without surgical resection for stage III non-small-cell lung cancer: a phase III randomised controlled trial. Lancet 2009;374:379-86.

4. Aupérin A, Le Pechoux C, Rolland E, et al. Meta-analysis of concomitant versus sequential radiochemotherapy in locally advanced non - small-cell lung cancer. J Clin Oncol 2010;28:2181-90.

5. Bradley JD, Paulus R, Komaki R, et al. Standard-dose versus high-dose conformal radiotherapy with concurrent and consolidation carboplatin plus paclitaxel with or without cetuximab for patients with stage 3 NSCLC RTOG 0617 - Bradley Lancet 2015. Lancet Oncol 2015;16:187-99.

6. Machtay M, Bae K, Movsas B, et al. Higher biologically effective dose of radiotherapy is associated with improved outcomes for locally advanced non-small cell lung carcinoma treated with chemoradiation: An analysis of the radiation therapy oncology group. Int J Radiat Oncol Biol Phys 2012;82:425-34.

7. Curran WJ, Paulus R, Langer CJ, et al. Sequential vs concurrent chemoradiation for stage iii non-small cell lung cancer: Randomized phase III trial RTOG 9410. J Natl Cancer Inst 2011;103:1452-60.

8. Milton DT, Miller VA. Advances in cytotoxic chemotherapy for the treatment of metastatic or recurrent non-small cell lung cancer. Semin Oncol 2005;32:299-314.

9. Noble J, Ellis PM, Mackay JA, et al. Second-line or subsequent systemic therapy for recurrent or progressive non-small cell lung cancer: A systematic review and practice guideline. J Thorac Oncol 2006;1:1042-58.

10. Reck M, Rodríguez-Abreu D, Robinson AG, et al. Pembrolizumab versus Chemotherapy for PD-L1Positive Non-Small-Cell Lung Cancer. N Engl J Med 
2016;375:1823-33.

11. Suntharalingam M, Paulus R, Edelman MJ, et al. Radiation Therapy Oncology Group Protocol 02-29: A Phase II Trial of Neoadjuvant Therapy With Concurrent Chemotherapy and Full-Dose Radiation Therapy Followed by Surgical Resection and Consolidative Therapy for Locally Advanced Non-small Cell Carcinoma of. Int J Radiat Oncol Biol Phys 2012;84:456-63.

12. Vyfhuis MAL, Bhooshan N, Burrows WM, et al. Oncological outcomes from trimodality therapy receiving definitive doses of neoadjuvant chemoradiation ( $\geq 60 \mathrm{~Gy}$ ) and factors influencing consideration for surgery in stage III non-small cell lung cancer. Adv Radiat Oncol 2017;2:259-69.

13. Green N, Melbye RW. Lung cancer: Retreatment of local recurrence after definitive irradiation. Cancer 1982;49:865-8.

14. Albain KS, Rusch VW, Crowley JJ, et al. Concurrent cisplatin/etoposide plus chest radiotherapy followed by surgery for stages IIIA (N2) and IIIB non-small-cell lung cancer: mature results of Southwest Oncology Group phase II study 8805. J Clin Oncol 1995;13:1880-92.

15. Farray D, Mirkovic N AK, Albain KS. Multimodality therapy for stage III non-small-cell lung cancer. J Clin Oncol 2005;23:3257-69.

16. Eberhardt WE, Albain KS, Pass H, et al. Induction treatment before surgery for non-small cell lung cancer. Lung Cancer 2003;42 Suppl 1:S9-14.

17. Jeremić B, Videtic GMM. Chest reirradiation with external beam radiotherapy for locally recurrent nonsmall-cell lung cancer: A review. Int J Radiat Oncol Biol Phys 2011;80:969-77.

18. Jackson MA, Ball DL. Palliative retreatment of locallyrecurrent lung cancer after radical radiotherapy 1987;147:391-4.

19. McAvoy S, Ciura K, Wei C, et al. Definitive reirradiation for locoregionally recurrent non-small cell lung cancer with proton beam therapy or intensity modulated radiation therapy: Predictors of high-grade toxicity and survival outcomes. Int J Radiat Oncol Biol Phys 2014;90:819-27.

20. McAvoy SA, Ciura KT, Rineer JM, et al. Feasibility of proton beam therapy for reirradiation of locoregionally recurrent non-small cell lung cancer. Radiother Oncol 2013;109:38-44.

21. Chao HH, Berman AT, Simone CB 2nd, et al. MultiInstitutional Prospective Study of Reirradiation with Proton Beam Radiotherapy for Locoregionally Recurrent Non-Small Cell Lung Cancer. J Thorac Oncol
2017;12:281-92.

22. Okamoto Y, Murakami M, Yoden E, et al. Reirradiation for locally recurrent lung cancer previously treated with radiation therapy. Int J Radiat Oncol Biol Phys 2002;52:390-6.

23. Wu KL, Jiang GL, Qian H, et al. Three-dimensional conformal radiotherapy for locoregionally recurrent lung carcinoma after external beam irradiation: A prospective phase I-II clinical trial. Int J Radiat Oncol Biol Phys 2003;57:1345-50.

24. Tada T, Fukuda H, Matsui K, et al. Non-small-cell lung cancer: Reirradiation for loco-regional relapse previously treated with radiation therapy. Int J Clin Oncol 2005;10:247-50.

25. Chun SG, Hu C, Choy H, et al. Impact of IntensityModulated Radiation Therapy Technique for Locally Advanced Non-Small-Cell Lung Cancer: A Secondary Analysis of the NRG Oncology RTOG 0617 Randomized Clinical Trial. J Clin Oncol 2017;35:56-62.

26. Speirs CK, DeWees TA, Rehman S, et al. Heart Dose Is an Independent Dosimetric Predictor of Overall Survival in Locally Advanced Non - Small Cell Lung Cancer. J Thorac Oncol 2017;12:293-301.

27. Graham MV, Purdy JA, Emami B, et al. Clinical dosevolume histogram analysis for pneumonitis after 3D treatment for non-small cell lung cancer (NSCLC). Int J Radiat Oncol Biol Phys 1999;45:323-9.

28. Kong FM, Ten Haken RK, Schipper MJ et al. Highdose radiation improved local tumor control and overall survival in patients with inoperable/unresectable nonsmall-cell lung cancer: long-term results of a radiation dose escalation study. Int J Radiat Oncol Biol Phys 2005;63:324-33.

29. Boyle J, Ackerson B, Gu L, et al. Dosimetric advantages of intensity modulated radiation therapy in locally advanced lung cancer. Adv Radiat Oncol 2017;2:6-11.

30. Galvin JM, Ezzell G, Eisbrauch A, et al. Implementing IMRT in clinical practice: A joint document of the American Society for Therapeutic Radiology and Oncology and the American Association of Physicists in Medicine. Int J Radiat Oncol Biol Phys 2004;58:1616-34.

31. Liao ZX, Komaki RR, Thames HD, et al. Influence of Technologic Advances on Outcomes in Patients With Unresectable, Locally Advanced Non-Small-Cell Lung Cancer Receiving Concomitant Chemoradiotherapy. Int J Radiat Oncol Biol Phys 2010;76:775-81.

32. Diwanji TP, Mohindra P, Vyfhuis M, et al. Advances in radiotherapy techniques and delivery for non-small cell 
lung cancer: Benefits of intensity-modulated radiation therapy, proton therapy, and stereotactic body radiation therapy. Transl Lung Cancer Res 2017;6:131-47.

33. Fleckenstein J, Eschler A, Kremp K, et al. Dose distribution and tumor control probability in out-of-field lymph node stations in intensity modulated radiotherapy (IMRT) vs 3D-conformal radiotherapy (3D-CRT) of nonsmall-cell lung cancer: an in silico analysis. Radiat Oncol 2015;10:178.

34. Beavis AW, Abdel-Hamid A, Upadhyay S. Re-treatment of a lung tumour using a simple intensity-modulated radiotherapy approach. Br J Radiol 2005;78:358-61.

35. Kruser TJ, McCabe BP, Mehta MP, et al. Reirradiation for locoregionally recurrent lung cancer: outcomes in small cell and non-small cell lung carcinoma. Am J Clin Oncol 2014;37:70-6.

36. Verma V, Rwigema JM, Malyapa RS, et al. Systematic assessment of clinical outcomes and toxicities of proton radiotherapy for reirradiation. Radiother Oncol 2017;125:21-30.

37. Simone CB 2nd, Rengan R. The use of proton therapy in the treatment of lung cancers. Cancer J 2014;20:427-32.

38. Auberger T, Seydl K, Futschek T, et al. Photons or Protons: Precision Radiotherapy of Lung Cancer. Strahlenther Onkol 2007;183:3-6.

39. Liu H, Chang JY. Proton therapy in clinical practice. Chin J Cancer 2011;30:315-26.

40. Register SP, Zhang X, Mohan R, et al. Proton stereotactic body radiation therapy for clinically challenging cases of centrally and superiorly located stage i non-small-cell lung cancer. Int J Radiat Oncol Biol Phys 2011;80:1015-22.

41. Zhang X, Li Y, Pan X, et al. Intensity-Modulated Proton Therapy Reduces the Dose to Normal Tissue Compared With Intensity-Modulated Radiation Therapy or Passive Scattering Proton Therapy and Enables Individualized Radical Radiotherapy for Extensive Stage IIIB NonSmall-Cell Lung Canc. Int J Radiat Oncol Biol Phys 2010;77:357-66.

42. Kraus KM, Heath E, Oelfke U. Dosimetric consequences of tumour motion due to respiration for a scanned proton beam. Phys Med Biol 2011;56:6563-81.

43. Lomax AJ. Intensity modulated proton therapy and its sensitivity to treatment uncertainties 2 : the potential effects of inter-fraction and inter-field motions. Phys Med Biol 2008;53:1043-56.

44. Chang JY, Jabbour SK, De Ruysscher D, et al. Consensus Statement on Proton Therapy in Early-Stage and Locally Advanced Non-Small Cell Lung Cancer. Int J Radiat
Oncol Biol Phys 2016;95:505-16.

45. Kang M, Huang S, Solberg TD, et al. A study of the beam-specific interplay effect in proton pencil beam scanning delivery in lung cancer. Acta Oncol 2017;56:531-40.

46. Seco J, Robertson D, Trofimov A, et al. Breathing interplay effects during proton beam scanning: simulation and statistical analysis. Phys Med Biol 2009;54:N283-94.

47. Dowdell S, Grassberger C, Sharp GC, et al. Interplay effects in proton scanning for lung: a 4D Monte Carlo study assessing the impact of tumor and beam delivery parameters. Phys Med Biol 2013;58:4137-56.

48. Grassberger C, Dowdell S, Lomax A, et al. Motion interplay as a function of patient parameters and spot size in spot scanning proton therapy for lung cancer. Int J Radiat Oncol Biol Phys 2013;86:380-6.

49. Kang Y, Zhang X, Chang JY, et al. 4D Proton treatment planning strategy for mobile lung tumors. Int J Radiat Oncol Biol Phys 2007;67:906-14.

50. Liu W, Liao Z, Schild SE, et al. Impact of Respiratory Motion on Worst-Case-Scenario Optimized IntensityModulated Proton Therapy for Lung Cancers. Pract Radiat Oncol 2015;5:e77-86.

51. Chang JY, Li H, Zhu XR, et al. Clinical implementation of intensity modulated proton therapy for thoracic malignancies. Int J Radiat Oncol Biol Phys 2014;90:809-18.

52. Chang JY, Zhang X, Knopf A, et al. Consensus Guidelines for Implementing Pencil-Beam Scanning Proton Therapy for Thoracic Malignancies on Behalf of the PTCOG Thoracic and Lymphoma Subcommittee. Int J Radiat Oncol Biol Phys 2017;99:41-50.

53. Ho JC, Nguyen QN, Li H, et al. Reirradiation of thoracic cancers with intensity modulated proton therapy. Pract Radiat Oncol 2017:S1879-8500(17)30196-0.

54. Badiyan SN, Mohindra P, Larson GL, et al. Clinical Outcomes of Patients With Recurrent Lung Cancer Reirradiated with Proton Therapy on the Proton Collaborative Group Prospective Registry Trial. Int J Radiat Oncol Biol Phys 2017;99:S209.

55. Timmerman R, Paulus R, Galvin J, et al. Stereotactic body radiation therapy for inoperable early stage lung cancer. JAMA 2010;303:1070-6.

56. Onishi H, Araki T, Shirato H, et al. Stereotactic hypofractionated high-dose irradiation for stage I nonsmall cell lung carcinoma: Clinical outcomes in 245 subjects in a Japanese multiinstitutional study. Cancer 2004;101:1623-31.

57. Baumann P, Nyman J, Hoyer M, et al. Outcome in a prospective phase II trial of medically inoperable 
stage I non-small-cell lung cancer patients treated with stereotactic body radiotherapy. J Clin Oncol 2009;27:3290-6.

58. Videtic GMM, Donington J, Giuliani M, et al. Stereotactic body radiation therapy for early-stage non-small cell lung cancer: Executive Summary of an ASTRO Evidence-Based Guideline. Pract Radiat Oncol 2017;7:295-301.

59. Simone CB 2nd, Dorsey JF. Additional data in the debate on stage I non-small cell lung cancer: surgery versus stereotactic ablative radiotherapy. Ann Transl Med 2015;3:172.

60. Zhang J, Yang F, Li B, et al. Which is the optimal biologically effective dose of stereotactic body radiotherapy for stage i non-small-cell lung cancer? A meta-analysis. Int J Radiat Oncol Biol Phys 2011;81:e305-16.

61. Wulf J, Baier K, Mueller G, et al. Dose-response in stereotactic irradiation of lung tumors. Radiother Oncol 2005;77:83-7.

62. Norihisa Y, Nagata Y, Takayama K, et al. Stereotactic Body Radiotherapy for Oligometastatic Lung Tumors. Int J Radiat Oncol Biol Phys 2008;72:398-403.

63. Baumann BC, Nagda SN, Kolker JD, et al. Efficacy and safety of stereotactic body radiation therapy for the treatment of pulmonary metastases from sarcoma: A potential alternative to resection. J Surg Oncol 2016;114:65-9.

64. Timmerman RD, Kavanagh BD, Cho LC, et al. Stereotactic body radiation therapy in multiple organ sites. J Clin Oncol 2007;25:947-52.

65. Nyman J, Hallqvist A, Lund J, et al. SPACE - A randomized study of SBRT vs conventional fractionated radiotherapy in medically inoperable stage I NSCLC. Radiother Oncol 2016;121:1-8.

66. Chang JY, Balter PA, Dong L, et al. Stereotactic Body Radiation Therapy in Centrally and Superiorly Located Stage I or Isolated Recurrent Non-Small-Cell Lung Cancer. Int J Radiat Oncol Biol Phys 2008;72:967-71.

67. Kelly P, Balter PA, Rebueno N, et al. Stereotactic body radiation therapy for patients with lung cancer previously treated with thoracic radiation. Int J Radiat Oncol Biol Phys 2010;78:1387-93.

68. Peulen H, Karlsson K, Lindberg K, et al. Toxicity after reirradiation of pulmonary tumours with stereotactic body radiotherapy. Radiother Oncol 2011;101:260-6.

69. Kilburn JM, Kuremsky JG, Blackstock AW, et al. Thoracic re-irradiation using stereotactic body radiotherapy (SBRT) techniques as first or second course of treatment. Radiother Oncol 2014;110:505-10.
70. Liu H, Zhang X, Vinogradskiy YY, et al. Predicting radiation pneumonitis after stereotactic ablative radiation therapy in patients previously treated with conventional thoracic radiation therapy. Int J Radiat Oncol Biol Phys 2012;84:1017-23.

71. Meijneke TR, Petit SF, Wentzler D, et al. Reirradiation and stereotactic radiotherapy for tumors in the lung: Dose summation and toxicity. Radiother Oncol 2013;107:423-7.

72. Parks J, Kloecker G, Woo S, et al. Stereotactic Body Radiation Therapy as Salvage for Intrathoracic Recurrence in Patients With Previously Irradiated Locally Advanced Non-Small Cell Lung Cancer. Am J Clin Oncol 2016;39:147-53.

73. Patel NR, Lanciano R, Sura K, et al. Stereotactic body radiotherapy for re-irradiation of lung cancer recurrence with lower biological effective doses. J Radiat Oncol 2015;4:65-70.

74. Reyngold M, Wu AJ, McLane A, et al. Toxicity and outcomes of thoracic re-irradiation using stereotactic body radiation therapy (SBRT). Radiat Oncol 2013;8:99.

75. Seung SK. Salvage SBRT for Previously Irradiated Lung Cancer. J Cancer Ther 2011;2:190-5.

76. Trovo M, Minatel E, Durofil E, et al. Stereotactic body radiation therapy for re-irradiation of persistent or recurrent non-small cell lung cancer. Int J Radiat Oncol Biol Phys 2014;88:1114-9.

77. Trakul N, Harris JP, Le QT, et al. Stereotactic Ablative Radiotherapy for Reirradiation of Locally Recurrent Lung Tumors. J Thorac Oncol 2012;7:1462-5.

78. Bezjak A, Paulus R, Gaspar LE, et al. Efficacy and Toxicity Analysis of NRG Oncology/RTOG 0813 Trial of Stereotactic Body Radiation Therapy (SBRT) for Centrally Located Non-Small Cell Lung Cancer (NSCLC). Int J Radiat Oncol Biol Phys 2016;96:S8.

79. Kramer GWPM, Gans S, Ullmann E, et al. Hypofractionated external beam radiotherapy as retreatment for symptomatic non-small-cell lung carcinoma: An effective treatment? Int J Radiat Oncol Biol Phys 2004;58:1388-93.

80. Montebello JF, Manatunga A, Horvath JL, et al. The reirradiation of recurrent bronchogenic carcinoma with external beam irradiation. Am J Clin Oncol 1993;16:482-8.

81. Gressen EL, Werner-Wasik M, Cohn J, et al. Thoracic Reirradiation for Symptomatic Relief After Prior Radiotherapeutic Management for Lung Cancer. Am J Clin Oncol 2000;23:160-3.

82. Schuurbiers OC, Kaanders JH, van der Heijden HF, et al. The PI3-K/AKT-Pathway and Radiation Resistance 
Mechanisms in Non-small Cell Lung Cancer. J Thorac Oncol 2009;4:761-7.

83. Kesarwala AH, Ko CJ, Ning H, et al. Intensity-modulated proton therapy for elective nodal irradiation and involvedfield radiation in the definitive treatment of locally advanced non-small-cell lung cancer: A dosimetric study. Clin Lung Cancer 2015;16:237-44.

84. Lin L, Kang M, Huang S, et al. Beam specific planning target volumes incorporating 4DCT for pencil beam scanning proton therapy of thoracic tumors. J Appl Clin Med Phys 2015;16:281-92.

85. Timmerman R, McGarry R, Yiannoutsos C, et al. Excessive toxicity when treating central tumors in a phase II study of stereotactic body radiation therapy for medically inoperable early-stage lung cancer. J Clin Oncol 2006;24:4833-9.

86. Chang JY, Zhang W, Komaki R, et al. Long-term outcome of phase I/II prospective study of dose-escalated proton therapy for early-stage non-small cell lung cancer. Radiother Oncol 2017;122:274-80.

87. Brahmer J, Reckamp KL, Baas P, et al. Nivolumab versus Docetaxel in Advanced Squamous-Cell Non-Small-Cell Lung Cancer. N Engl J Med 2015;373:123-35.

88. Borghaei H, Paz-Ares L, Horn L, et al. Nivolumab versus Docetaxel in Advanced Nonsquamous Non-Small-Cell

Cite this article as: Vyfhuis MA, Rice S, Remick J, Mossahebi S, Badiyan S, Mohindra P, Simone CB 2nd. Reirradiation for locoregionally recurrent non-small cell lung cancer. J Thorac Dis 2018;10(Suppl 21):S2522-S2536. doi: 10.21037/jtd.2017.12.50
Lung Cancer. N Engl J Med 2015;373:1627-39.

89. Antonia SJ, Villegas A, Daniel D, et al. Durvalumab after Chemoradiotherapy in Stage III Non-Small-Cell Lung Cancer. N Engl J Med 2017:NEJMoa1709937.

90. Herbst RS, Baas P, Kim DW, et al. Pembrolizumab versus docetaxel for previously treated, PD-L1-positive, advanced non-small-cell lung cancer (KEYNOTE-010): A randomised controlled trial. Lancet 2016;387:1540-50.

91. Tang C, Wang X, Soh H. Combining Radiation and Immunotherapy: A New Systemic Therapy for Solid tumors? Cancer Immunol Res 2014;2:831-8.

92. Kalbasi A, June CH, Haas N, et al. Radiation and immunotherapy: A synergistic combination. J Clin Invest 2013;123:2756-63.

93. Kang J, Demaria S, Formenti S. Current clinical trials testing the combination of immunotherapy with radiotherapy. J Immunother Cancer 2016;4:51.

94. Jabbour SK, Berman AT, Simone CB 2nd. Integrating immunotherapy into chemoradiation regimens for medically inoperable locally advanced non-small cell lung cancer. Transl Lung Cancer Res 2017;6:113-8.

95. Simone CB 2nd, Burri SH, Heinzerling JH. Novel radiotherapy approaches for lung cancer: combining radiation therapy with targeted and immunotherapies. Transl lung cancer Res 2015;4:545-52. 